Relations industrielles

Industrial Relations

\title{
La fusion du C.M.T. et du C.C.T.
}

\section{Gérard Dion}

Volume 10, numéro 2, mars 1955

URI : https://id.erudit.org/iderudit/1022746ar

DOI : https://doi.org/10.7202/1022746ar

Aller au sommaire du numéro

Éditeur(s)

Département des relations industrielles de l'Université Laval

ISSN

0034-379X (imprimé)

1703-8138 (numérique)

Découvrir la revue

Citer ce document

Dion, G. (1955). La fusion du C.M.T. et du C.C.T. Relations industrielles /

Industrial Relations, 10(2), 135-138. https://doi.org/10.7202/1022746ar

Tous droits réservés (C Département des relations industrielles de l’Université Laval, 1955
Ce document est protégé par la loi sur le droit d'auteur. L’utilisation des services d'Érudit (y compris la reproduction) est assujettie à sa politique d'utilisation que vous pouvez consulter en ligne.

https://apropos.erudit.org/fr/usagers/politique-dutilisation/ 
Dautre part, la technique est encore à notre époque en pleine évolution. Les conclusions auxquelles est parvenu le Congrès international des Ingénieurs catholiques dans le domaine des relations humaines dans lentreprise devront donc sans cesse être reprises et approfondies en fonction de l'évolution des techniques, afin que le progrès technique et économique ne soit pas un obstacle au progrès humain, mais au contraire contribue à l'épanouissement total de lhomme, fils de Dieu et frère du Christ.

\section{FAITS ET COMMENTAIRES}

- Monsieur Robert-E. Heneault, surintendant des relations industrielles à The Steel Company of Canada, Limited, Montréal, poursuit une série de cours aux étudiants du Département des relations industrielles.

- Monsieur L. Hemsworth, gérant des relations industrielles à la Canadian Industries Limited, est également professeur invité au Département des relations industrielles.

- Monsieur Gérard Dion, prêtre, directeur-adjoint du Département des relations industrielles, professeur à l'Université Laval a prononcé au Séminar de l'Association des Marchands Détaillants, temu à l'Université de Montréal le 4 avril dernier, une conférence intitulée \&Syndicalisme patronal et syndicalisme ouvrier »; Monsieur Dion a également été invité à donner devant l'Ecole ouvrière de Shawinigan une causerie sur l'intégration du syndicalisme dans la province de Québec.

- Monsieur Emile Gosselin, secrétaire du Département des relations industrielles, professeur à l'Université Laval a été en janvier dernier, conférencier invité de la Ligue des Propriétaires de Québec; le sujet traité fut celui de l'administration municipale;

Monsieur Gosselin donna le 26 février une conférence intitulée - \& Service au public par la collaboration devant les membres de la Corporation des électriciens de la province de Québec et les membres du bureau des examinateurs;

Monsieur Gosselin prononça une causerie sur la convention collective devant. PAssociation patronale des services hospitaliers.

\section{LA FUSION DU CMT ET DU CCT}

Les quotidiens ont déjà présenté à la population de notre pays l'importante nouvelle de la fusion probable des deux grandes centrales syndicales canadiennes avant l'hiver prochain. Ce geste suit de près une mesure qui a été prise aux EtatsUnis entre la FAT et le CIO. Il fallait s'y attendre, car l'unité étant créée aux Etats-Unis, il n'y avait plus de raison pour qu'elle ne soit pas réalisée au Canada.

Nous n'avons pas le temps d'expliquer en long et en large les causes qui avaient amené la division de ces forces ouvrières ni les conséquences que la fusion va entraîner sur le syndicalisme canadien. Cependant parce que déjà des interprétations folichonnes ont été données à cet évènement nous croyons nécessaire de faire quelques remarques essentielles. 


\section{Aux Etats-Unis}

1. On a attribué toutes sortes de raisons à la scission au sein de la FAT en 1935. Pour plusieurs la cause déterminante était due à une divergence au sujet des formules d'organisation syndicale. Les traditionnalistes de la FAT voulant s'en tenir strictement aux unions de métiers et les progressistes soutenant les syndicats industriels. Aujourd'hui tout le monde s'accorde à dire que malgré l'importance de cette question, ce ne fut qu'une occasion. La scission a été principalement une affaire de personnes et lacteur fut John Lewis. Dès 1936 des tentatives sérieuses d"unification ont été proposées, mais John Lewis les a fait avorter.

2. Malgré certains bienfaits concrets, cette scission dans le syndicalisme américain a toujours été considérée par les catholiques experts en sciences sociales comme un malheur et ils ont toujours préconisé le retour à l'unité d'organisation et d'action. Aujourd'hui ils se réjouissent de l'évènement. Aucune raison de principe ni de technique ne s'opposait à cette fusion. En effet il est normal que dans toute centrale syndicale se rencontrent des chefs à tendances progressistes et d'autres à tendances conservatrices. C'est un signe de dynamisme et de démocratie. La poursuite d'un équilibre est avantageuse aux travailleurs et à la nation. Parmi les unions affiliées à la FAT il y en eut toujours qui étaient organisées sur une base industrielle.

3. La disparition de tous les personnages qui avaient joué un rôle actif lors de la scission (John Lewis a peu près retiré de la scène syndicale et ne faisant plus partie de la FAT ni du CIO, Philip Murray et William Green décédés); la nécessité de faire un front commun pour syndiquer les travailleurs non encore organisés; la résistance à opposer à une administration anti-sympathique à la cause syndicale. Voilà les trois raisons qui ont immédiatement amené la réalisation d'un voeu partagé par les travailleurs américains et presque tous leurs chefs.

\section{Av CaNaDa}

Il n'y eut pas de raisons fondamentales pour la création du CCT au Canada en 1940. Les querelles de personnes ne sont pas entrées en ligne de compte. Un groupement national désigné sous le nom de Congrès pan-canadien du travail existait déjà à côté du CMT et au point de vue orientation se rapprochait des Trade Unions d'Angleterre. Le CMT, à la demande de la FAT a expulsé de ses rang les locaux canadiens des unions affiliées au CIO aux Etats-Unis. Ces locaux, n'appartenant plus à aucune centrale canadienne ont décidé de s'entendre avec le Congrès pan-canadien et de former le CCT. Les deux unions qui ont joué un rôle de premier plan dans cette transformation du Congres pan-canadien en Congres Canadien du Travail furent la Canadian Brotherhood of Railway Employees (Mosher) et l'Union des Mineurs de la Nouvelle-Ecosse (Conroy). Dès 1941 le CCT réunissait 120,000 membres alors que le CMT en avait 144,000.

L'orientation du CMT est restée la même. Dans l'ensemble un groupement à tendance conservatrice et pragmatique officiellement opposé à laction politique directe. Quant au CCT il est plus jeune, plus dynamique, favorable à laction politique directe non-partisane. Il ne veut pas se confondre avec le parti CCF, mais il le supporte parce que de tous les partis politiques canadiens, la CCF, est le seul à avoir accepté de promouvoir et de défendre les réclamations politiques du CCT.

En 1954, les effectifs combinés du CMT (596,004) et du CCT (360,782) forment 967,786 membres sur un total de 1,267,911 travailleurs syndiqués, soit $80 \%$ des syndiqués canadiens. La CTCC possède des effectifs s'élevant à 100,312 membres. 


\section{LES ÉTAPES DE LA FUSION}

1. Le principe de la fusion a été accepté par les congrès annuels du CMT et du CCT l'an dernier et chacune des centrales a désigné des mandataires pour négocier un plan de fusion. Ce comité formé de représentants du CMT et du CCT s'est réuni, s'est entendu sur certains points et a laissé d'autres à l'étude.

Les points importants déjà réglés sont les suivants: a) La création d'une nouvelle centrale syndicale autonome; b) ladmission dans cette centrale des unions affiliées au CMT et au CCT sur un pied d'égalité ainsi que des autres groupements syndicaux « légitimes » (entendre non-communistes); c) les structures régionales et départementales de chaque centrale sont maintenues et les employés conservent leur emploi; d) les fédérations provinciales et conseils locaux de chaque centrale seront fusionnés dès que possible.

2. L'entente agréée par le comité de fusion n'a pas de valeur actuellement. Elle doit être ratifiée par l'assemblée annuelle du CMT qui aura lieu à la fin de mai et celle du CCT qui se tiendra en octobre 1955. Si les deux congrès ratifient le projet, une assemblée générale de représentants des deux centrales se réunira pour fonder la nouvelle centrale. Ce que lon attend en 1956.

\section{EN QUOI CONSISTE LA FUSION}

A strictement parler, la fusion du CMT et du CCT n'est pas une fusion complète.

Pour qu'il y ait fusion complète, il faudrait une amalgamation de tous les organismes de chacune des centrales à tous les paliers. Or il ny a d'almalgamation que sur le plan supérieur. A l'intérieur de la nouvelle centrale co-existent les mêmes unions qu'autrefois. Ainsi l'union du textile affiliée au CMT contimuera à exister à côté de celle qui possède la même juridiction dans la CCT: l'une et l'autre demeureront autonomes. L'Association internationale des machinistes (CMT) et les Métallos-unis d'Amérique, (CCT) conserveront leur autonomie, etc. Pas plus que dans le passé, la centrale nouvelle n'aura autorité sur les groupements qui lui seront affiliés et pourra leur imposer des directives.

La concurrence syndicale qui existe actuellement sur le plan industriel et sur le plan des usines ne disparaîtra pas. Elle pourra être réduite selon un code d'éthique professionnelle suggéré aux unions, mais que celles-ci seront libres d'accepter ou de refuser. Néanmoirs, en vertu des clauses de «non-piratage», les deux groupes se sont entendus pour décourager lenvahissement d'un secteur par une union rivale.

En somme il n'y aura de fusion que sur le plan supérieur pour fin de représentation (non de négociation) vis-à-vis du gouvernement et du public. L'action économique des syndicats par rapport aux employeurs n'est pas du domaine de la centrale syndicale, mais de celui des unions et de leurs locaux. Or ces derniers ne sont pas fusionnés.

Pour le moment disons que cete fusion est loin de réaliser un monopole syndical ou un «One big union», ainsi que le soutiennent de tout le poids de leur ignorance les profanes en relations industrielles.

\section{LES CONSÉQUENCES DE LA FUSION}

Bien que cette fusion soit limitée à un palier bien précis, les conséquences qu'elle entraînera seront considérables dans le domaine politique et dans le domaine syndical. 
1. La puissance de représentation de cette nouvelle centrale devant le public canadien et le gouvernement fédéral, sera accrue et indirectement celle des autres centrales, si elles demeurent étrangères à se mouvement, sera diminuée pour autant, sinon annihilée.

2. Dans lorganisation des travailleurs qui sont actuellement non-syndiqués, les possibilites d'extension des centrales indépendantes seront certainement diminuées, sinon rendues très problématiques.

3. Les groupements régionaux qui n'adhéreront pas à la fusion ne sont pas nécessairement voués à la disparition. L'expérience a prouvé que tout en demeurant indépendants. de tels groupements peuvent se maintenir à condition de trouver une forme de collaboration sur des bases permanentes.

4. Si dans le projet de fusion, on prévoit pour l'avenir l'amalgamation des unions du CMT et du CCT qui possèdent la mème juridiction, il nous semble qu'à cause de difficultés quasi insurmontables dues à Thistoire, la tradition, aux rivatités de personnes et à la volonté mème des travailleurs concernés, on est en face d'une ébauche assez proche de lutopie. A moins que le régime démocratique actuel fasse place à un autre, à notre sens, la nouvelle centrale syndicale devra se résoudre encore longtemps à accepter et conserver dans son sein des unions concurrentes comme cela se pratique en Angleterre dans le Trade Union Congress. Cette formule d'ailleurs non seulement est plus réaliste, mais elle comporte l'avantage de mieux sauvegarder la liberté des travailleurs à opter pour une union de leur chaix.

GÉRARD DION

\section{LIVRES et REVUES}

Baker, H. and France, R., Centralization and Decentralization in Industrial Relations, Industrial Relations Section, Princeton University, $210 \mathrm{pp}$., 1954.

Voilà un travail bien fait dont lordre et la concision sont sùrement les deux meilleures qualités. Les objectifs recherchés, l'étendue du travail, les méthodes employées ainsi que le contenu de certaines exoressions sont suffisamment précisés dès le début pour que le lecteur puisse apprécier la valeur et la portée des conclusions auxquelles les auteurs arrivent.

En second lien, les informations accumulkes au cours de l'enquête sont nettement classees sous deux rubriques principales a) la localisation des responsabilités quant da la formulation et lapplication dune politique générale en ce qui concerne le personnel; b) la loca- lisation de ces mêmes responsabilités en ce qui a trait à certaines fonctions spécifiques comme Intilisation de la maind'oeuvre, Ja détermination des auditions de travail ou l'elaboration des programmes de sécurité de l'employé

Subsequente à la formation d'un plan irtelligent, la facilité avec laquelle les différentes parties du volume s'intègrent est une invitation à whe lecture qui porte des fruits doubles: en mème temps quil éclaire ces connaissances sur un problème particulier, le lecteur trouve lexemple d'une étude bien conduite.

Le volume est intéressant à d'autres points de vue. En effet lanalyse du partage des responsabilités quant à la détermination des obiectifs et à leur réalisation est un problème qui transcende la gestion du personnel, de telle sorte que bon nombre de remarques faites li l'occasion de cette dernière peuvent être utiles lorsque sont envisagées la gestion financière ou technique de l'entreprise.

De plus, les résultats de lenquête faite dans 135 compagnies dont la maind'oenvre globale dépassait $3,500,000$ sont 